\title{
Applying State-of-the-Art Survival Extrapolation Techniques to the Evaluation of CAR-T Therapies: Evidence from a Systematic Literature Review
}

\author{
Matthew Sussman (D) - Concetta Crivera · Jennifer Benner • \\ Nicholas Adair
}

Received: May 6, 2021 / Accepted: June 22, 2021 / Published online: July 12, 2021

(C) The Author(s) 2021

\section{ABSTRACT}

Introduction: Traditional statistical techniques for extrapolating short-term survival data for anticancer therapies assume the same mortality rate for noncured and "cured" patients, which is appropriate for projecting survival of non-curative therapies but may lead to an underestimation of the treatment effectiveness for potentially curative therapies. Our objective was to ascertain research trends in survival extrapolation techniques used to project the survival benefits of chimeric antigen receptor $\mathrm{T}$ cell (CAR-T) therapies.

Methods: A global systematic literature search produced a review of survival analyses of CAR-T therapies, published between January 1, 2015 and December 14, 2020, based on publications sourced from MEDLINE, scientific conferences, and health technology assessment agencies. Trends in survival extrapolation techniques used, and the rationale for selecting advanced techniques, are discussed.

Supplementary Information The online version contains supplementary material available at https:// doi.org/10.1007/s12325-021-01841-4.

M. Sussman $(\varangle) \cdot J$. Benner $\cdot$ N. Adair Panalgo LLC, 265 Franklin Street, Suite 1101, Boston, MA 02110, USA

e-mail: msussman@panalgo.com

C. Crivera

Janssen Scientific Affairs LLC, Titusville, NJ, USA
Results: Twenty publications were included, the majority of which $(65 \%, N=13)$ accounted for curative intent of CAR-T therapies through the use of advanced extrapolation techniques, i.e., mixture cure models [MCMs] $(N=10)$ or spline-based models $(N=3)$. The authors' rationale for using the MCM approach included (a) better statistical fits to the observed Kaplan-Meier curves (KMs) and (b) visual inspection of the KMs indicated that a proportion of patients experienced long-term remission and survival which is not inherently captured in standard parametric distributions.

Discussion: Our findings suggest that an advanced extrapolation technique should be considered in base case survival analyses of CAR-T therapies when extrapolating short-term survival data to long-term horizons extending beyond the clinical trial duration.

Conclusion: Advanced extrapolation techniques allow researchers to account for the proportion of patients with an observed plateau in survival from clinical trial data; by only using standard-partitioned modeling, researchers may risk underestimating the survival benefits for the subset of patients with long-term remission. Sensitivity analysis with an alternative advanced extrapolation technique should be implemented and re-assessment using clinical trial extension data and/or real-world data should be conducted as longer-term data become available. 
Keywords: CAR-T therapies; Extrapolation; Kaplan-Meier; Mixture cure; Survival analysis; Survival modeling

\section{Key Summary Points}

Why carry out this study?

Partitioned-survival modeling, via standard parametric survival functions, has been historically common in survival analyses of anticancer therapies; however, several advanced extrapolation techniques (e.g., mixture cure models, spline-based models) have been established and implemented to account for the potentially curative nature of novel treatments, including chimeric antigen receptor $\mathrm{T}$ cell (CAR-T) therapies.

Given the different statistical methods available to researchers, the goal of this research was to conduct a global systematic literature review to identify trends and rationale in survival extrapolation techniques used when projecting the survival benefits of CAR-T therapies over time.

\section{What was learned from this study?}

Our findings suggest that nearly twothirds of cost-effectiveness (CE) models (65\%) used advanced extrapolation techniques in base case analyses to account for the potentially curative nature of CAR-T therapies for a proportion of treated patients.

On the basis of this observation, it is our position that future survival analyses of CAR-T therapies should consider incorporating these advanced techniques in base case analyses when extrapolating short-term survival data from clinical trials to long-term horizons that extend well beyond the clinical trial duration.

\section{DIGITAL FEATURES}

This article is published with digital features, including a summary slide, to facilitate understanding of the article. To view digital features for this article go to https://doi.org/10.6084/ m9.figshare.14823333.

\section{INTRODUCTION}

Five chimeric antigen receptor (CAR) T cell therapies have been approved by the United States (US) Food and Drug Administration (FDA) for relapsed or refractory (R/R) diffuse large B cell lymphoma (DLBCL) (axicabtagene ciloleucel ["axi-cel"; Yescarta] [1], tisagenlecleucel ["tis-cel"; Kymriah] [2], lisocabtagene maraleucel [Breyanzi] [3]), B cell precursor acute lymphoblastic leukemia (ALL) (tis-cel) [2], R/R mantle cell lymphoma (brexucabtagene autoleucel [Tecartus] [4]), and most recently idecabtagene vicleucel (Abecma) for R/R multiple myeloma [5]. Additionally, one further CAR-T therapy is in development for $\mathrm{R} / \mathrm{R}$ multiple myeloma (ciltacabtagene autoleucel ["ciltacel"]) $[6,7]$, while several others are in earlier stage development for hematologic cancers and solid tumors [8]. The approved CAR-T therapies as well as those therapies in development have the potential to cure a proportion of patients receiving these novel agents, with "cure" statistically defined as the observed plateauing of survival data (i.e., progression-free survival [PFS], overall survival [OS]) some years after diagnosis [9-11].

Industry stakeholders including public and private payers, manufacturers, academics, health service researchers, and health technology assessment (HTA) agencies have a vested interest in monitoring the value of new CAR-T therapies over time. For anticancer therapies, clinical value is often measured in terms of survival benefits defined by incremental gains in life expectancy (i.e., life years [LYs]) compared to alternative sources of care. To estimate clinical value, industry researchers rely on the best available clinical data (i.e., PFS, OS, response rates) at the time of analysis. Mature clinical data with years of follow-up duration, 
obtained either through clinical trial extensions or real-world data following product launch and uptake, offer a robust method for estimating survival benefits given the observational nature of the long-term data. In particular, findings from phase 3 clinical trials are important for confidently confirming the survival benefit identified in short-term and/or single cohort phase 2 clinical trials. However, in the absence of mature or confirmatory clinical data, industry researchers project survival benefits beyond the clinical trial duration by using various statistical methods to extrapolate short-term survival data to longer periods including the patient's lifetime.

Partitioned-survival modeling, via standard parametric survival functions, has often been used to extrapolate short-term survival data (PFS, OS) of anticancer therapies [12]. Traditional techniques for estimating survival assume the same mortality rate for all patients, which is appropriate for anticancer therapies that extend life but do not necessarily offer the potential for cure. However, assuming one shared value for mortality across "cured" and noncured patients may lead to an underestimation of the effectiveness of treatment for potentially curative therapies [13]. In response to this potential limitation to standard parametric survival estimation, researchers have explored several advanced extrapolation techniques to account for the potentially curative nature of novel treatments, including CAR-T therapies. For instance, cure fraction models, including the mixture cure fraction model (or mixture cure model [MCM]) and the non-mixture cure fraction model (or non-mixture cure model [NMCM], have been used as an alternative approach to modeling the heterogeneity between long-term survivors (i.e., cured patients) and those who are not (i.e., non-cured patients) $[14,15]$. MCMs assume that a proportion of patients are cured (i.e., long-term survivors) and thus are not at risk of experiencing the event of interest (e.g., progression or death), while assuming that the remaining proportion of non-cured patients will eventually experience the event of interest $[9,10]$. In this regard, cured patients are assigned background mortality rates similar or equal to the general population while non-cured patients are assigned an additional disease-specific mortality risk. NMCMs are based on the assumption that, following anticancer therapy, cancer cells may remain in the body and may slowly grow over time producing a relapse of disease; techniques of this type allow the researcher to scale the survival curve until the plateau (e.g., cure fraction) is reached [16]. Another example of an advanced extrapolation technique includes flexible parametric or spline models which do not explicitly introduce a cure fraction in the way that a cure approach would; instead, patients who are still alive after a particular point in time (e.g., 5 years) are assumed to be effectively cured and have a similar or equivalent mortality to the general population for the remainder of the model horizon $[17,18]$. The point at which patients are deemed to be cured are termed "knots." Goodness-of-fit statistics (i.e., Akaike information criterion [AIC], Bayesian information criterion [BIC]) indicate how many knots, and at which time point, fit best to the primary intervention's OS and PFS data.

Depending on the extrapolation technique used, an intervention's survival benefit may substantially vary. In an analysis of long-term survival and cost-effectiveness (CE) associated with axi-cel for the treatment of B cell lymphoma, Whittington et al. used five different survival extrapolation techniques, including standard parametric, flexible parametric, two mixture cure, and flexible parametric mixture extrapolation techniques, to project short-term survival data from clinical trials to a lifetime horizon [19]. The authors found that incremental LYs ranged from 1.89 to 5.82; the smallest difference in LYs was found using the standard parametric approach while the largest difference in LYs was found using an MCM approach, suggesting an underestimation of incremental LYs using traditional techniques. Similarly, Bansal et al. compared survival outcomes using standard parametric techniques to an MCM approach among patients treated with axi-cel for R/R large B cell lymphoma [20]. The authors determined that the use of standard survival models yielded overall survival estimates of 2.0 years (Weibull distribution) and 3.0 years (generalized gamma distribution) 
compared to 15.7 years using MCMs, providing further evidence that traditional extrapolation techniques have the potential to underestimate projections on long-term survival.

In addition to the availability of different statistical options for researchers, there appears to be little consensus in the literature, along with a lack of statistical guidelines from professional societies, regarding which statistical method should be used to extrapolate survival of potentially curative therapies. As a result, it is important to ascertain research trends in survival extrapolation methods for the purpose of advancing the field of comparative effectiveness research of CAR-T therapies. Doing so will aid in creating a consistent model framework for use and interpretation by industry stakeholders when evaluating a CAR-T therapy's long-term value. Consistency in statistical analytics is a critical consideration since different extrapolation techniques yield different model results and different product valuations, which could have downstream impacts on payer formulary decisions and patient access to care. While some publications have reviewed and compared survival extrapolation techniques for immunooncologic therapies [13, 21-31], none have evaluated survival extrapolation trends across the full range of publications (i.e., peer-reviewed or conference proceedings) nor global HTA agencies specifically for CAR-T therapies. To this end, the goal of this research was to conduct a systematic literature review of analyses projecting the survival benefits of CAR-T therapies over time, in an effort to ascertain research trends in survival extrapolation techniques used and the rationale for selecting advanced techniques.

\section{METHODS}

A global systematic literature search was conducted to identify trends in survival extrapolation techniques associated with estimating the survival benefits of CAR-T therapies. The review was conducted in accordance with PRISMA and CHEERS guidelines for systematic literature reviews [32, 33]. Publications were sourced from MEDLINE (PubMed), scientific conference databases and publications, and health technology assessment (HTA) agencies. A comprehensive description of the full set of search terms used for this study can be found in Tables 1-3 in the supplementary material.

PubMed was searched from January 1, 2015 to December 14, 2020; the start date of January 1, 2015 was selected to allow for inclusion of all relevant survival extrapolation-based analyses published in support of CAR-T therapies as the first CAR-T therapy was approved in the USA in 2017. The search terms implemented in PubMed included a combination of keywords and MeSH terms related to CAR-T therapies and analyses that predominantly utilize survival extrapolation techniques; for this reason, focus was given to comparative effectiveness research relating to $\mathrm{CE}$ analyses. Included studies were limited to those written in the English language (or with an available English translation) and with the availability of full text via PubMed filters. Overall inclusion criteria required studies to evaluate CAR-T therapies in a human population, incorporate a modeling component such as Markov cycles or survival curve extrapolation, be an economic evaluation and/or use LYs or QALYs as part of the primary outcome (e.g., incremental cost per LY gained). Additionally, $\mathrm{CE}$ analyses that do not extrapolate clinical trial data beyond the maximum follow-up duration were deemed inapplicable to this qualitative evaluation of extrapolation techniques used.

In addition, hematologic and health economic and outcomes research conferences were searched from 2018 to 2020. Conferences included the American Society of Hematology (ASH) Annual Meeting, European Hematology Association (EHA) Annual Meeting, American Society of Clinical Oncology (ASCO) Annual Meeting and Virtual Scientific Meetings, and all International Society of Pharmacoeconomics and Outcomes Research (ISPOR) meetings. Abstracts and posters were included in our detailed review provided that information concerning the survival extrapolation technique (e.g., MCM, spline-based, standard parametric) was specified.

Finally, targeted searches of the HTAs were conducted to identify the most prominent and relevant CE models from agencies such as the 
National Institute for Health and Care Excellence (NICE) (UK), Canadian Agency for Drugs and Technologies in Health (CADTH) (Canada), Medical Services Advisory Committee (MSAC) (Australia), Independent Institute for Quality and Efficiency in Health Care (IQWIG) (Germany), and Institute for Clinical and Economic Review (ICER) (US), as well as any other CE models that were recommended by industry experts. Global HTA bodies are often at the forefront of methodological techniques for the purpose of comparative effectiveness, and CE, analyses since reimbursement decisions often rely on their guidance. Evaluating the methods employed and/or endorsed by global HTA bodies is critical to identifying methodological trends in this area. Assessments were included if they evaluated a CAR-T therapy, regardless of publication date. Key search terms included "chimeric antigen", "tisagenlecleucel", "axicabtagene", "lisocabtagene", and "brexucabtagene"; ongoing assessments of these therapies were excluded since the extrapolation technique(s) applied in the analyses have not yet been described. To avoid the risk of including studies twice, peer-reviewed publications were excluded if an HTA was available.

Detailed data were extracted regarding the survival extrapolation approach (e.g., MCM, spline-based), OS parametric distribution (e.g., Weibull, exponential, log-normal), clinical trial used for extrapolation and the corresponding follow-up duration, model time horizon, interventions, comparators, and how treatment efficacy translates to clinical measures (i.e., incremental LYs). These findings were organized and assessed to determine commonalities in methodological choices and innovations; results are presented across the compilation of peer-reviewed (i.e., publications, conference proceedings) and non-peer-reviewed studies (i.e., HTA evaluations) as well as separately for each.

This article is based on previously conducted studies and does not contain any new studies with human participants or animals performed by any of the authors; the article therefore did not require ethics committee approval or patient consent.

\section{RESULTS}

\section{Overview}

A total of 78 titles were returned using the search terms, of which 65 were identified as fulfilling the relevant search criteria (e.g., full text, English, within the search time horizon) and 17 were identified as having relevant titles and abstracts. After a comprehensive review of each peer-reviewed publication, seven studies were identified as meeting our inclusion criteria. From the HTA review, 65 HTAs were returned using the search terms and 15 HTAs were identified as relevant on the basis of a review of titles. After a full review of each HTA, nine were identified as meeting our search criteria. From the conference abstract review, 916 posters were returned using the search terms. After a full review of each abstract and/or poster, four were identified as meeting our search criteria and having extractable data (e.g., survival extrapolation approach, OS survival function). An additional four conference abstracts were identified as meeting our search criteria; however, these abstracts did not contain extractable data. Twenty publications were included in this literature review. Figure 1 provides an attrition flowchart of the study selection methodology.

One study, Lin 2019 [adult], evaluated both axi-cel and tis-cel in separate models within the same publication [34]. Similarly, the ICER evaluation of B cell cancers consisted of separate evaluations of tis-cel for pediatric B-ALL and axi-cel for adult DLBCL [35]. The authors of ICER's HTA evaluation also published their methods and results as two standalone publications in peer-reviewed journals which were excluded from this review $[19,36]$. For the purposes of this analysis, ICER's assessment was counted among the HTA evaluations. A total of 22 CE analyses were evaluated in 20 publications.

Of the 20 published CE models reviewed, six were conducted from the US perspective (Lin et al. [pediatric] [37], Lin et al. [adult] [34], Roth et al. [38], Sarkar et al. [39], Liu et al. [40], ICER [35]), three each from the UK (NICE [ALL] [41], 


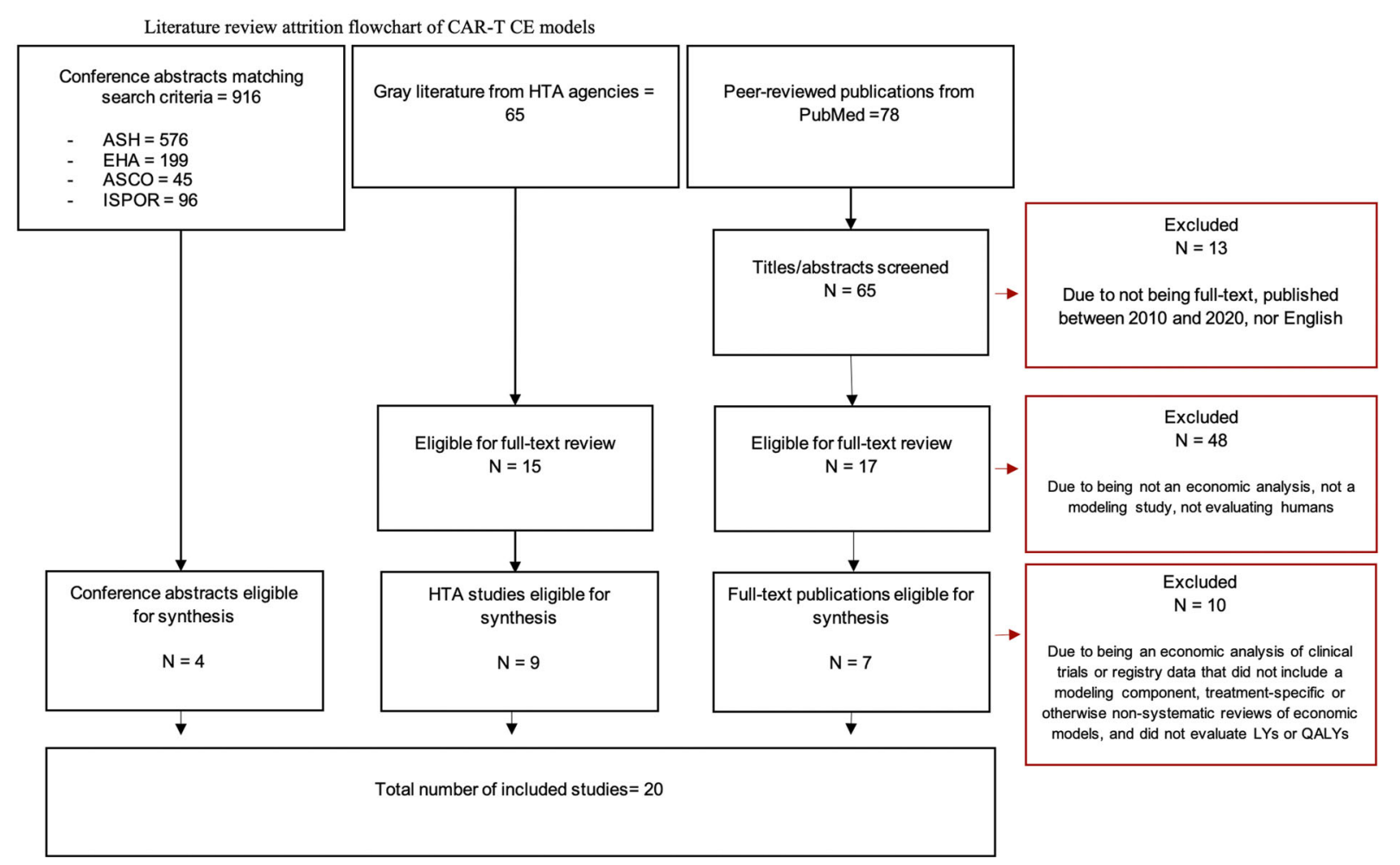

Fig. 1 Literature review attrition flowchart of CAR-T CE models

NICE [Axi] [42], NICE [Tis] [43]), Canadian (Furzer et al. [44], CADTH [Axi] [45], CADTH [Tis] [46]), and Australian perspectives (MSAC [ALL] [47], MSAC [Axi] [48], MSAC [Tis] [49]), two from the Spanish perspective (Ribera Santasusana et al. [50], Sierra et al. [51]), and one each from the Dutch (Thielen et al. [52]), Italian (Marchetti et al. [53]), Swedish, Nordic, Finnish, and Denmark perspectives (Karampampa et al. [54]).

All publications were studied in pediatric B-ALL or adult DLBCL. Table 1 summarizes the key modeling techniques, clinical trials, interventions, comparators, and incremental clinical outcomes (i.e., incremental LYs) for each model.

\section{Survival Modeling Techniques}

The majority of CE models $(80 \%, N=16 / 20)$ estimated the proportion of patients per health state from partitioned-survival models, in which model selection was based on the best- fitting parametric model for each PFS and OS dataset $[35,38,40-43,45-54]$. Of the $20 \mathrm{CE}$ models, $13(65 \%, \quad N=13 / 20)$ accounted for curative intent of the primary CAR-T intervention through the use of advanced extrapolation techniques, including mixture cure or splinebased models, in base case analyses. Of the remaining seven models, three employed a standard partitioned-survival modeling approach in base case analyses $[35,47,52]$ and four employed alternative methods for estimating the proportion of patients per health state (i.e., microsimulation, optimization) $[34,37,39,44]$. When stratifying the results according to peer-reviewed and non-peer-reviewed status, our findings indicate a higher proportion of non-peer-reviewed studies used advanced techniques $(78 \%, N=7 / 9)$ compared to peer-reviewed studies $(55 \%, N=6 / 11)$.

CE models assessing axi-cel generally used ZUMA-1 data with a median follow-up time of 27.1 months [55], except for Karampampa et al. [54], which used ZUMA-1 data with a median follow-up time of 39.1 months. CE models 


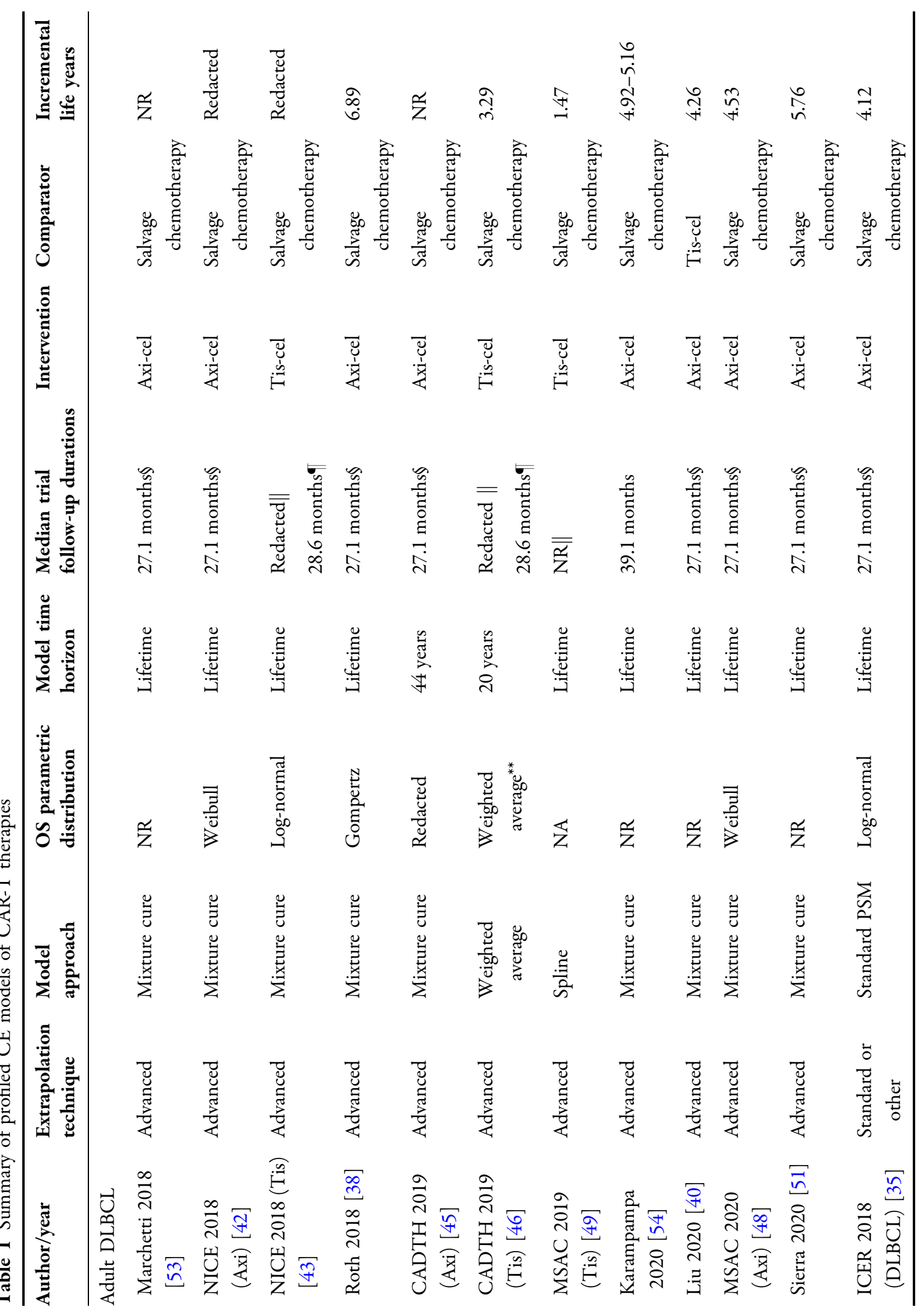




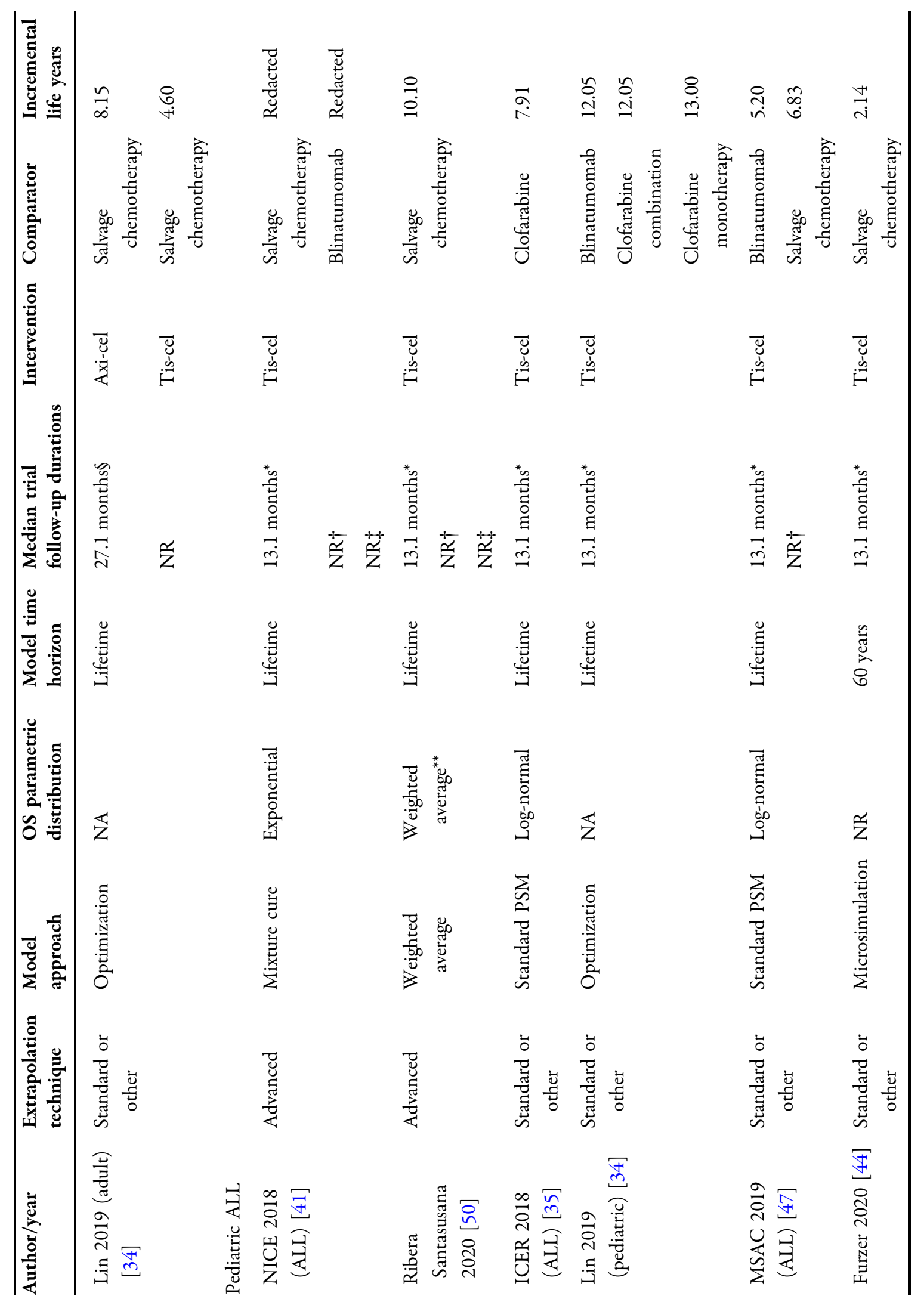




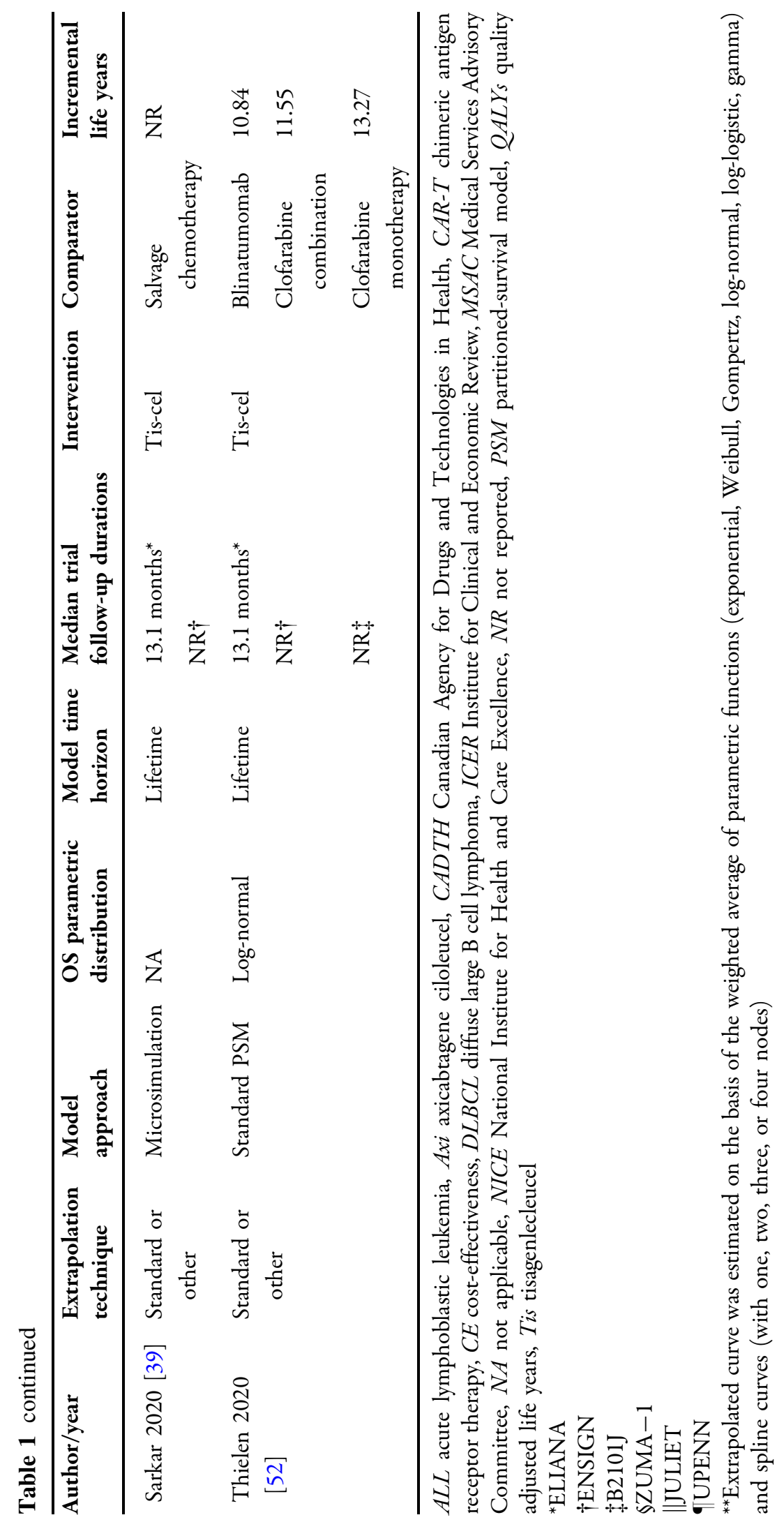


assessing tis-cel in pediatric B-ALL based extrapolations on the ELIANA and ENSIGN clinical trials; ELIANA reported median followup times of 13.1 months and ENSIGN did not report a median follow-up time [56, 57]. CE models assessing tis-cel in adult DLBCL based extrapolations on the JULIET trial, which did not report a median follow-up time, and the UPenn trial, which reported a median follow-up time of 28.6 months [58].

\section{Mixture Cure or Spline-Based Models}

Ten CE models utilized a mixture cure approach, while three CE models utilized a spline-based approach. Only four of the mixture cure models reported the OS parametric distribution (exponential, Weibull, log-normal, Gompertz) [38, 41-43]; the majority of CE models had the parametric distribution redacted or did not report one. In CE models for pediatric B-ALL, tis-cel was compared to clofarabine monotherapy, clofarabine combination therapy, blinatumomab, and salvage chemotherapy. In CE models for adult DLBCL, axi-cel and tis-cel were compared to standard of care (SOC) (e.g., salvage chemotherapy, best supportive care, best available therapy); Liu et al. compared axi-cel and tis-cel head-to-head.

In justifying the mixture cure modeling approach, authors of the aforementioned CE models noted that the mixture cure approach provided better statistical fits to the observed Kaplan-Meier curves compared to traditional parametric fits based on AIC and BIC criteria $[38,41-43]$. In addition, the authors noted that visual inspection of the observed Kaplan-Meier curves for PFS and OS indicated plateaus of PFS and OS, providing rationale that a proportion of patients experienced long-term (e.g., "durable") remission and survival, which is not inherently captured in standard parametric distributions [38, 41-43, 45].

HTAs noted that there were a number of caveats to the application of mixture cure approaches in survival extrapolations. First, they noted that the robust estimates of MCMs require two key elements: (1) data from studies with long follow-up times that far exceed the anticipated point of cure time, and (2) sufficient numbers of patients at risk at the end of follow- up in order to robustly estimate a cure fraction. Second, they noted two key risks: (1) extrapolation methods may overestimate the benefits of the CAR-T therapy and underestimate the benefits of the comparator therapy, and (2) the short follow-up may preclude the possibility of late relapses that could not have been captured in the extrapolation of OS data.

Ribera Santasusana et al., the authors of CADTH's evaluation of tis-cel in adult DLBCL, and the authors of MSAC's evaluation of tis-cel all employed survival techniques in which a spline model was employed, or a weighted average of parametric distributions and spline models was taken instead of using one parametric distribution over another. In Ribera Santasusana et al., the authors explained that no curve precisely matched the observed Kaplan-Meier curves from the respective clinical trials based on AIC and BIC criteria; thus, the authors applied methods from Jackson et al., in which a weighted average of parametric models and splines is taken on the basis of the AIC criteria [59]. The authors of the MSAC assessment explained that the spline extrapolation provided a better statistical fit to the observed Kaplan-Meier data than the log-normal extrapolation method, which was considered to be overly conservative and to underestimate the survival benefit of tis-cel.

\section{Standard Partitioned-Survival Models or Other Modeling Techniques}

Of the 20 CE models reviewed, three employed standard partitioned-survival modeling approaches and four employed alternative methods (i.e., microsimulation, optimization) [34, 35, 37, 47, 52]. In CE models applying standard approaches, authors stated that the parametric distributions were based on goodness-of-fit statistics (e.g., AIC, BIC) and a visual comparison of the extrapolations to the observed Kaplan-Meier curves, which were confirmed by a clinical expert $[35,52]$. In CE models which employed a microsimulation approach, authors argued that the microsimulation framework allowed for a more complex model design, including the ability to track individual patient's history, compared to traditional Markov CE models and partitioned- 
survival models [39]. In the two optimization CE models included in the review, Lin et al. argued that standard partitioned-survival models may not be entirely appropriate for modeling multiple lines of therapy; instead, Lin et al. explained that they adopted a Markov CE model framework in which they implemented an optimization approach to estimating PFS and OS; under this optimization approach, the authors were able to parameterize the separate rates of progression, pre-progression mortality, and post-progression mortality, and incorporate differential outcomes according to initial response and/or transplantation recipient, as well as time-varying costs and utilities [34, 37].

In addition, there were four conference abstracts which applied standard partitionedsurvival modeling techniques to evaluations of CAR-T therapies; however, these studies did not provide information on the OS parametric distribution utilized, clinical trial used for extrapolation, or median trial follow-up duration, yielding them unfit for profiling in our analysis [60-63].

\section{DISCUSSION}

\section{Implications for Future Survival Modeling of CAR-T Therapies}

There is a lack of consensus in the literature, along with a lack of statistical guidelines from professional societies, regarding which statistical method should be used to extrapolate survival of potentially curative therapies. As a result, this study sought to elicit trends in survival extrapolation techniques used in analyses of CAR-T therapies, in particular, through a systematic review of CE models that apply this type of statistical methodology. Contrary to other reviews and syntheses, this qualitative assessment was unique in its evaluation of a wider body of evidence with a broader focus on publications in peer-reviewed journals, health technology appraisals for regional HTA agencies, and conference proceedings. Moreover, this review focused exclusively on CAR-T therapies given the abundance of these novel agents in early-phase development for both hematologic cancers and solid tumors. This study involved a review of which statistical techniques were chosen for implementation, and while our analysis did not involve a comparison of how or when to select a specific technique, our evidence synthesis should provide guidance and direction for future survival analyses of CAR-T therapies in development.

Our findings suggest that nearly two-thirds of CE models (65\%) used advanced extrapolation techniques in base case analyses to account for the potentially curative nature of CAR-T therapies for a proportion of treated patients. Moreover, a higher proportion of non-peer-reviewed (i.e., HTA evaluations; 78\%) used advanced techniques compared to peer-reviewed publications (55\%). Although sample sizes were small, this is an important finding since global HTA bodies are responsible for reimbursement guidance and inaccurate estimation of the survival benefit of new therapies could lead to inappropriate HTA guidance. On the basis of the overall and stratified observations, it is our position that future survival analyses of CAR-T therapies should consider incorporating these techniques when extrapolating short-term survival data from clinical trials to long-term horizons that extend well beyond the clinical trial duration. Although partitioned-survival modeling, via standard parametric survival functions, has been historically common in survival analyses of anticancer therapies, the curative intent of novel CAR-T treatments has pushed researchers to re-evaluate the most appropriate extrapolation techniques. Advanced extrapolation techniques, such as MCMs and flexible parametric/splinebased models, provide greater flexibility to account for the proportion of patients with an observed plateau in PFS and/or OS from clinical trial data than standard partitioned-survival modeling [9]; by only using standard-partitioned modeling, researchers risk underestimating the survival benefits for the subset of patients with long-term remission $[13,20,30,64]$. 


\section{Extrapolation Techniques for Other Immuno-Oncology Therapies}

Several publications investigated the applicability of standard partitioned-survival and advanced extrapolation techniques for immuno-oncology therapies, other than CARTs, by comparing modeled survival projections from early extracts of clinical trial data to actual survival from more mature clinical trial data. Estimates of survival and goodness-of-fit statistics were compared across the alternative approaches. The identified studies indicated that survival estimates derived from advanced extrapolation techniques better aligned with clinical expectations and provided better fits to the more mature, observed clinical trial data.

For instance, Bullement et al. compared different predicted estimates of OS based on standard and spline-based parametric approaches using four different data extracts from the JAVELIN Merkel 200 (JM200) trial of avelumab for patients with metastatic Merkel cell carcinoma; each extract varied according to the minimum follow-up available for all patients in the trial. The authors found that spline-based models provided the best statistical goodnessof-fit scores as well as a more accurate estimate of OS using longer-term trial data [29]. Similarly, Bullement et al. compared projections from the piecewise survival approach used in NICE's evaluation of ipilimumab for melanoma (TA319) and from advanced extrapolation techniques (i.e., spline-based models, mixture models, MCMs) to actual survival from a longerterm clinical trial extract (i.e., 5-year follow-up) [65]. The authors found that the MCM, along with the survival approach used in TA319, more accurately predicted the observed 5-year survival data than standard parametric, spline, and non-curative mixture models which underestimated 5-year survival. Also, Ouwens et al. applied several extrapolation techniques (i.e., standard parametric, flexible parametric, cure, parametric mixture, response-based landmark models) to an early data extract from the ATLANTIC clinical trial of durvalumab for previously treated, advanced non-small cell lung cancer; the projections were compared to an updated extract from the ATLANTIC clinical trial [30]. The authors found that standard parametric models tended to underestimate long-term OS, and that cure, parametric mixture, and landmark models provided a better fit for the observed plateau in longer-term survival data. Moreover, Gibson et al. evaluated the applicability of standard parametric models and restricted cubic spline models among patients with metastatic melanoma; extrapolated data from the CheckMate 067 trial of nivolumab plus ipilimumab were validated using external PFS data for 5-year survival with ipilimumab monotherapy [64]. Similar to other analyses and comparisons, the authors found that standard parametric survival underestimated PFS and that restricted cubic spline models with one knot provided a better fit to the trial data especially at the plateau phase of the survival curve. While it is unclear whether the findings from these examples can be generalized to all cancer types including future indications of CAR-T therapies in development, these publications strengthen the argument that advanced extrapolation techniques should be considered when projecting survival data of immuno-oncology, and specifically CAR-T, therapies.

\section{Opportunities for Future Research}

The publications cited in the previous section provide examples of validating extrapolated survival data for immuno-oncology therapies, other than CAR-Ts, by comparing predicted estimates from shorter duration clinical trials to actual observations from longer-term clinical trials. Few such validation exercises have been conducted in support of CAR-T therapies, specifically. One validation of extrapolated survival data from the ZUMA-1 clinical trial of axi-cel (median follow-up of 15.4 months) was compared to a newer cut of ZUMA-1 trial data (median follow-up of 27.1 months) [21]. The authors concluded that MCMs better predicted Kaplan-Meier data available from the updated ZUMA-1 trial published in 2018 [55]. Following the presentation of this validation exercise, more recent ZUMA-1 trial data, with median follow-up of 39.1 months and with a median OS of 25.8 months, were presented at ASH 2020 
[66]. Continuing to re-evaluate the appropriateness of survival extrapolation techniques using the most recent clinical trial extension data will serve to strengthen survival analyses and its outputs. As a result, follow-on analyses to our qualitative assessment could compare extrapolated survival data from early clinical trial reports for axi-cel and tis-cel to observed survival from longer follow-up clinical trial reports $[66,67]$. In addition to extended clinical trial data, real-world observational data could also be used to confirm extrapolation techniques. However, despite the approvals of both axi-cel and tis-cel in the USA in 2017, publications of real-world evidence (RWE) evaluating long-term survival of these therapies are lacking. Among the RWE publications of axi-cel and tis-cel, follow-up duration was not long enough (median follow-up duration ranged from 4 to 24 months across publications) to compare extrapolated survival data from shorter duration clinical trials to RWE observations [68-75]. Moreover, repeated validations of extrapolated techniques using real-world data should be considered as those databases are updated over time [76].

\section{CONCLUSIONS}

On the basis of the CAR-T survival extrapolation trends identified in this systematic literature review, coupled with the validation exercises of survival extrapolation techniques of immunooncology therapies more broadly, we would recommend an advanced extrapolation technique be considered in base case analyses of future survival analyses of CAR-T therapies. Sensitivity analysis with an alternative advanced extrapolation technique should be implemented and re-assessment using clinical trial extension data and/or real-world data should be conducted as longer-term data become available.

\section{ACKNOWLEDGEMENTS}

Funding. Sponsorship for this research study and the Journal's Rapid Service Fee was funded by Janssen Pharmaceuticals, Inc.

Medical Writing, Editorial, and Other Assistance. The authors thank Mr. Silas Martin and Mr. Mike Ingham (Janssen Scientific Affairs LLC) for providing assistance with manuscript conceptualization.

Authorship. All named authors meet the International Committee of Medical Journal Editors (ICMJE) criteria for authorship for this article, take responsibility for the integrity of the work as a whole, and have given their approval for this version to be published.

Author Contributions. All authors contributed to the study conception and design. Material preparation, data collection and analysis were performed by Matthew Sussman, Jennifer Benner, and Nicholas Adair. The first draft of the manuscript was written by Matthew Sussman, Jennifer Benner, and Nicholas Adair and all authors commented on previous versions of the manuscript. All authors read and approved the final manuscript.

Disclosures. Matthew Sussman, Jennifer Benner, and Nicholas Adair are employees of Panalgo LLC and were paid consultants in connection with the study. Concetta Crivera is an employee of Johnson \& Johnson ( $\&$ J) and has in excess of $\$ 10,000$ of $\mathrm{J} \& \mathrm{~J}$ stock.

Compliance with Ethics Guidelines. This article is based on previously conducted studies and does not contain any new studies with human participants or animals performed by any of the authors; the article therefore did not require ethics committee approval or patient consent.

Data Availability. This article involved a systematic review of the literature; therefore, all data and materials are publicly available. 
Open Access. This article is licensed under a Creative Commons Attribution-NonCommercial 4.0 International License, which permits any non-commercial use, sharing, adaptation, distribution and reproduction in any medium or format, as long as you give appropriate credit to the original author(s) and the source, provide a link to the Creative Commons licence, and indicate if changes were made. The images or other third party material in this article are included in the article's Creative Commons licence, unless indicated otherwise in a credit line to the material. If material is not included in the article's Creative Commons licence and your intended use is not permitted by statutory regulation or exceeds the permitted use, you will need to obtain permission directly from the copyright holder. To view a copy of this licence, visit http://creativecommons.org/licenses/by$\mathrm{nc} / 4.0 /$.

\section{REFERENCES}

1. Food \& Drug Administration. YESCARTA Prescribing Information. 2020. https://www.fda.gov/ media/108377/download. Accessed 18 Dec 2020.

2. Food \& Drug Administration. KYMRIAH Prescribing Information. 2018. https://www.fda.gov/files/ vaccines $\% 2$ C $\% 20$ blood $\% 20 \% 26 \% 20$ biologics/ published/Package-Insert-KYMRIAH.pdf. Accessed 18 Dec 2020

3. Food \& Drug Administration. BREYANZI Prescribing Information. 2021. https://www.fda.gov/ media/145711/download. Accessed 30 Mar 2021.

4. Food \& Drug Administration. Tecartus Prescribing Information. 2021. https://www.fda.gov/media/ 140409/download. Accessed 30 Mar 2021.

5. Food \& Drug Administration. Abecma Prescribing Information. 2021. https://www.fda.gov/media/ 147055/download. Accessed 19 Apr 2021.

6. Madduri D, Berdeja JG, Usmani SZ, et al. CARTITUDE-1: phase $1 \mathrm{~b} / 2$ study of ciltacabtagene autoleucel, a B cell maturation antigen-directed chimeric antigen receptor T cell therapy, in relapsed/refractory multiple myeloma. Blood. 2020;136(Supplement 1):22-5.

7. Munshi NC, Anderson LD, Shah N, et al. Idecabtagene vicleucel in relapsed and refractory multiple myeloma. N Engl J Med. 2021;384(8):705-16.
8. Hildreth C. CAR-T cell companies proliferate: list of CAR-T companies worldwide. BioInformant. 2021 Feb 3. https://bioinformant.com/car-t-companiesthe-meteoric-rise-of-cellular-immunotherapies/. Accessed 30 Mar 2021.

9. Othus M, Barlogie B, LeBlanc ML, Crowley JJ. Cure models as a useful statistical tool for analyzing survival. Clin Cancer Res. 2012;18(14):3731-6.

10. Lambert PC, Thompson JR, Weston CL, Dickman PW. Estimating and modeling the cure fraction in population-based cancer survival analysis. Biostatistics. 2007;8(3):576-94.

11. Andersson TM-L, Lambert PC. Fitting and modeling cure in population-based cancer studies within the framework of flexible parametric survival models. Stata J. 2012;12(4):623-38.

12. Woods B, Sideris E, Palmer S, Latimer N, Soares M. NICE DSU technical support document 19: partitioned survival analysis for decision modelling in health care: a critical review report by the decision support unit. 2017. Available from http://www. nicedsu.org.uk.

13. Othus M, Bansal A, Koepl L, Wagner S, Ramsey S. Accounting for cured patients in cost-effectiveness analysis. Value Health. 2017;20(4):705-9.

14. Boag JW. Maximum likelihood estimates of the proportion of patients cured by cancer therapy. J R Stat Soc Ser B Methodol. 1949;11(1):15-53.

15. Tsodikov AD, Ibrahim JG, Yakovlev AY. Estimating cure rates from survival data: an alternative to twocomponent mixture models. J Am Stat Assoc. 2003;98(464):1063-78.

16. Yakovlev A, Asselain B, Bardou V, et al. A simple stochastic model of tumor recurrence and its applications to data on pre-menopausal breast cancer. In: Biometrics and analysis dormees spatio-temporal. Rennes: French Society of Biometrics; 1993.

17. Lambert PC, Royston P. Further development of flexible parametric models for survival analysis. Stata J Promot Commun Stat Stata. 2009;9(2): 265-90.

18. Nelson CP, Lambert PC, Squire IB, Jones DR. Flexible parametric models for relative survival, with application in coronary heart disease. Stat Med. 2007;26(30):5486-98.

19. Whittington MD, McQueen RB, Ollendorf DA, et al. Long-term survival and cost-effectiveness associated with axicabtagene ciloleucel vs chemotherapy for treatment of B cell lymphoma. JAMA Netw Open. 2019;2(2):e190035. 
20. Bansal A, Sullivan SD, Lin VW, et al. Estimating long-term survival for patients with relapsed or refractory large B cell lymphoma treated with chimeric antigen receptor therapy: a comparison of standard and mixture cure models. Med Decis Mak. 2019;39(3):294-8.

21. Kasle A, Qu T, Meng Y. Review and comparison of overall survival extrapolation in health technology assessments CAR-T therapies. In: ISPOR. Orlando, FL, USA; 2020. https://www.ispor.org/docs/defaultsource/intl2020/icer-nice-os-methodspbi36-pdf. pdf?sfvrsn=f27cfaea_0. Accessed 16 Apr 2021.

22. Kroep S, Kiff C, Kraan C, Bianco M. Modelling the survival benefit of immuno-oncologic therapy: a review of methods used in NICE single technology appraisals. In: ISPOR Europe. Copenhagen, Denmark; 2019. https://www.pharmerit.com/wpcontent/uploads/2019/11/MODELING-THESURVIVAL-BENEFIT-OF-IMMUNO-ONCOLOGICTHERAPY-A-REVIEW-OF-METHODS-USED-INNICE-SINGLE-TECHNOLOGY-APPRAISALS.pdf. Accessed 16 Apr 2021.

23. Damuzzo V, Agnoletto L, Leonardi L, Chiumente M, Mengato D, Messori A. Analysis of survival curves: statistical methods accounting for the presence of long-term survivors. Front Oncol. 2019;4(9):453.

24. Ramsey S, Othus M, Roth J, Yuan Y, Wagner S, Penrod J. PRM126-a comparison of mixture cure and standard parametric modeling to estimate long-term survival for small-cell lung cancer in patients treated with nivolumab after progression. Value Health. 2018;21:S377.

25. Nukala U, Messan MR, Yogurtcu ON, Wang X, Yang H. A systematic review of the efforts and hindrances of modeling and simulation of CAR T cell therapy. ArXiv210105359 Q-Bio. 2021 Mar 2. http://arxiv. org/abs/2101.05359. Accessed 16 Apr 2021.

26. Foix Colonier A, Genestier V, Lueza B. PCN205 comparison of methods to account for cured patients in a cost-effectiveness analysis: a case study in oncology. Value Health. 2019;22:S475.

27. Porteous A, van Hest N, Griffiths M, Morten P. Exploring the use and acceptance of alternative survival modelling approaches for immuno-oncology therapies in UK Technology Assessments. In: ISPOR Europe. Barcelona, Spain; 2018. https:// www.costellomedical.com/wp-content/uploads/ 2018/11/Survival-Modelling.pdf. Accessed 16 Apr 2021.

28. Cooper M, Smith S, Williams T, Harding T, AguiarIbáñez R. PCN293 how successful are standard and flexible extrapolation methods for the long-term projection of overall survival compared to trial data subsequently made available for cancer immunotherapies? Value Health. 2020;23:S75.

29. Bullement A, Willis A, Amin A, Schlichting M, Hatswell AJ, Bharmal M. Evaluation of survival extrapolation in immuno-oncology using multiple pre-planned data cuts: learnings to aid in model selection. BMC Med Res Methodol. 2020;20(1):103.

30. Ouwens MJNM, Mukhopadhyay P, Zhang Y, Huang M, Latimer N, Briggs A. Estimating lifetime benefits associated with immuno-oncology therapies: challenges and approaches for overall survival extrapolations. Pharmacoeconomics. 2019;37(9):1129-38.

31. Gray J, Sullivan T, Latimer NR, et al. Extrapolation of survival curves using standard parametric models and flexible parametric spline models: comparisons in large registry cohorts with advanced cancer. Med Decis Mak. 2021;41(2):179-93.

32. Moher D, Liberati A, Tetzlaff J, Altman DG, Group TP. Preferred reporting items for systematic reviews and meta-analyses: the PRISMA statement. PLOS Med. 2009;6(7):e1000097.

33. Husereau D, Drummond M, Petrou S, et al. Consolidated health economic evaluation reporting standards (CHEERS) statement. Value Health. 2013;16(2):e1-5.

34. Lin JK, Muffly LS, Spinner MA, Barnes JI, Owens DK, Goldhaber-Fiebert JD. Cost effectiveness of chimeric antigen receptor $\mathrm{T}$ cell therapy in multiply relapsed or refractory adult large B cell lymphoma. J Clin Oncol. 2019;37(24):2105-19.

35. Institute for Clinical and Economic Review. Chimeric antigen receptor $\mathrm{T}$ cell therapy for $\mathrm{B}$ cell cancers: effectiveness and value. 2018. https://icer. org/wp-content/uploads/2020/10/ICER_CAR_T_ Final_Evidence_Report_032318.pdf. Accessed 8 Jul 2021.

36. Whittington MD, McQueen RB, Ollendorf DA, et al. Long-term survival and value of chimeric antigen receptor $\mathrm{T}$ cell therapy for pediatric patients with relapsed or refractory leukemia. JAMA Pediatr. 2018;172(12):1161.

37. Lin JK, Lerman BJ, Barnes JI, et al. Cost Effectiveness of chimeric antigen receptor $\mathrm{T}$ cell therapy in relapsed or refractory pediatric B cell acute lymphoblastic leukemia. J Clin Oncol. 2018;36(32): 3192-202.

38. Roth JA, Sullivan SD, Lin VW, et al. Cost-effectiveness of axicabtagene ciloleucel for adult patients with relapsed or refractory large B cell lymphoma in the United States. J Med Econ. 2018;21(12): $1238-45$. 
39. Sarkar RR, Gloude NJ, Schiff D, Murphy JD. Costeffectiveness of chimeric antigen receptor $\mathrm{T}$ cell therapy in pediatric relapsed/refractory B cell acute lymphoblastic leukemia. J Natl Cancer Inst. 2019;111(7):719-26.

40. Liu R, Snider T, Diakite I, Tempeleear S, Botteman M. Cost effectiveness of axicabtagene ciloleucel (AXI-CEL) and tisagenlecleucel (TISA-CEL) for adult patients with relapsed or refractory large B cell lymphoma (RR LBCL) in the US. In: Virtual European Hematology Association Congress. 2020.

41. Walton M, Sharif S, Hodgson R, Claxton L. Tisagenlecleucel-T for treating relapsed or refractory $B$ cell acute lymphoblastic leukaemia in people aged up to 25 years. National Institute for Health and Care Excellence; 2018.

42. National Institute for Health and Care Excellence. Axicabtagene ciloleucel for treating diffuse large B cell lymphoma and primary mediastinal B cell lymphoma after 2 or more systemic therapies [ID1115]. National Institute for Health and Care Excellence; 2018.

43. Corbett M, Duarte A, Walker S, Wright K. Tisagenlecleucel for treating relapsed or refractory diffuse large B cell lymphoma. National Institute for Health and Care Excellence; 2018.

44. Furzer J, Gupta S, Nathan PC, et al. Cost-effectiveness of tisagenlecleucel vs standard care in high-risk relapsed pediatric acute lymphoblastic leukemia in Canada. JAMA Oncol. 2020;6(3):393.

45. Canadian Agency for Drugs and Technologies in Health. Axicabtagene ciloleucel for diffuse large B cell lymphoma: economic review report. 2019. https://www.cadth.ca/sites/default/files/pdf/car-t/ ct0002-axicabtagene-ciloleucel-economic-reportredacted.pdf. Accessed 8 Jul 2021.

46. Canadian Agency for Drugs and Technologies in Health. Tisagenlecleucel for diffuse large B cell lymphoma: economic review report. 2019. https:// cadth.ca/sites/default/files/pdf/car-t/op0538tisagenlecleucel-economic-report-DLBCL-jan2019. pdf. Accessed 8 Jul 2021.

47. Medical Services Advisory Committee. Tisagenlecleucel (CTL019) for treatment of refractory CD19. positive leukaemia and lymphoma: Public Summary Document. 2019. http://www.msac.gov.au/ internet/msac/publishing.nsf/Content/BE2E1EB50 ED57442CA2581F4000C1723/\$File/1519-Final\%20 PSD-April\%202019_redacted.pdf. Accessed $8 \mathrm{Jul}$ 2021.

48. Medical Services Advisory Committee. Axicabtagene ciloleucel (CAR-T therapy) for the treatment of refractory or relapsed CD19-positive lymphoma:
Public Summary Document. 2020. http://www.msac. gov.au/internet/msac/publishing.nsf/Content/B5B78 0278B3A4B48CA2583C9001B80BB/\$File/1587\%20 Final\%20PSD\%20Nov\%2019_redacted.pdf. Accessed 8 Jul 2021.

49. Medical Services Advisory Committee. Tisagenlecleucel (CTL019) for treatment of relapsed or refractory diffuse large B cell lymphoma (DLBCL): Public Summary Document. 2019. http://www. msac.gov.au/internet/msac/publishing.nsf/Content/ A2B10F9A03293BC8CA2583CF001C7A4D/\$File/15 $19.1 \% 20$ Final\%20updated\%20PSD\%20Nov\%2019_ redacted.pdf. Accessed 8 Jul 2021.

50. Ribera Santasusana JM, de Andrés Saldaña A, García-Muñoz N, Gostkorzewicz J, Martínez Llinàs D, Díaz-de-Heredia C. Cost-effectiveness analysis of tisagenlecleucel in the treatment of relapsed or refractory B cell acute lymphoblastic leukaemia in children and young adults in Spain. Clin Outcomes Res CEOR. 2020;12:253-64.

51. Sierra J, Briones J, Calleja A, Camacho C. Axicabtagene ciloleucel for the management of patients with diffuse large B cell lymphoma and primary mediastinal large B cell lymphoma: an economic evaluation for Spain. In: Virtual European Hematology Association Congress. 2020.

52. Thielen FW, Dongen-Leunis A, Arons AMM, Ladestein JR, Hoogerbrugge PM, Uyl-de Groot CA. Cost-effectiveness of anti-CD19 chimeric antigen receptor $\mathrm{T}$ cell therapy in pediatric relapsed/refractory B cell acute lymphoblastic leukemia. A societal view. Eur J Haematol. 2020;105(2):203-15.

53. Marchetti M, Martelli E, Zinzani PL. Cost-effectiveness of axicabtagene ciloleucel for relapsed or refractory dffuse large B cell lymphoma in Italy. Blood. 2018;132(Supplement 1):4779-4779.

54. Karampampa K, Stene E, Axelsen F, et al. PPM3 costeffectiveness of axicabtagene ciloleucel (AXI-CEL) vs standard of care for adult patients with relapsed or refractory diffuse large B cell lymphoma in Sweden, Norway, Finland, and Denmark. Value Health. 2020;23:S687.

55. Locke FL, Ghobadi A, Jacobson CA, et al. Long-term safety and activity of axicabtagene ciloleucel in refractory large B cell lymphoma (ZUMA-1): a single-arm, multicentre, phase 1-2 trial. Lancet Oncol. 2019;20(1):31-42.

56. Maude SL, Laetsch TW, Buechner J, et al. Tisagenlecleucel in children and young adults with B cell lymphoblastic leukemia. N Engl J Med. 2018;378(5):439-48.

57. Novartis Pharmaceuticals. Study of efficacy and safety of CTL019 in pediatric all patients (ENSIGN). 
Clinicaltrials.com; 2020. https://clinicaltrials.gov/ ct2/show/NCT02228096. Accessed 16 Apr 2021.

58. Schuster SJ, Svoboda J, Chong EA, et al. Chimeric antigen receptor $\mathrm{T}$ cells in refractory B cell lymphomas. N Engl J Med. 2017;377(26):2545-54.

59. Jackson CH, Thompson SG, Sharples LD. Accounting for uncertainty in health economic decision models by using model averaging. J R Stat Soc Ser A Stat Soc. 2009;172(2):383-404.

60. Hao Y, Eldjerou L, Yang H, Qi C, Globe D. Costeffectiveness analysis of CTL019 for the treatment of pediatric and young adult patients with relapsed or refractory B cell acute lymphoblastic leukemia in the United States. Blood. 2017;130(Supp1):609.

61. Kattamis A, Avgitidou A, Chatzopoulos S, Markouri A. Cost-effectiveness of tisagenlecleucel in paediatric and young adult relapsed or refractory B cell acute lymphobastic leukaemia, from a Greek social security system perspective. In: ISPOR EU. Copenhagen, Denmark; 2019. https://www.ispor.org/ heor-resources/presentations-database/presentation/ euro2019-3119/97419. Accessed 16 Apr 2021.

62. Yang H, Qi C, Zhang J, El Ouagari K. Cost-effectiveness of tisagenlecleucel for adults with relapsed or refractory diffuse large B cell lymphoma: a Canadian societal perspective. Value Health. 2018;21:S44.

63. Yang H, Zhang J, Hampe M. Cost-effectiveness of tisagenlecleucel in pediatric and young adult patients with relapsed or refractory B cell acute lymphoblastic leukemia: a Canadian societal perspective. Value Health. 2018;21:S43.

64. Gibson E, Koblbauer I, Begum N, et al. Modelling the survival outcomes of immuno-oncology drugs in economic evaluations: a systematic approach to data analysis and extrapolation. Pharmacoeconomics. 2017;35(12):1257-70.

65. Bullement A, Latimer NR, Bell GH. Survival extrapolation in cancer immunotherapy: a validation-based case study. Value Health. 2019;22(3): 276-83.

66. Jacobson C, Locke FL, Ghobadi A, et al. Long-term survival and gradual recovery of B cells in patients with refractory large B cell lymphoma treated with axicabtagene ciloleucel (Axi-Cel). Blood. 2020;136(Supplement 1):40-2.

67. Jaeger U, Bishop MR, Salles G, et al. Myc expression and tumor-infiltrating $\mathrm{T}$ cells are associated with response in patients (Pts) with relapsed/refractory diffuse large $\mathrm{B}$ cell lymphoma (r/r DLBCL) treated with tisagenlecleucel in the Juliet Trial. Blood. 2020;136(Supplement 1):48-9.

68. Fitzgerald L, Kittai S, Nastoupil L, Waller A. Real world outcomes of elderly patients with relapsed/ refractory diffuse large B cell lymphoma treated with chimeric antigen receptor T cell therapy: an updated analysis. In: 62nd ASH Annual Meeting \& Exposition. Virtual; 2020. https://ash.confex.com/ ash/2020/webprogram/Paper143236.html. Accessed 19 Apr 2021.

69. Chong EA, Chong ER, Landsburg DJ, et al. AntiCD19 CAR-T for treatment of double expressor and double hit large B cell lymphomas: a single institution real-world analysis. Blood. 2020;136(Supplement 1):19-20.

70. Buecklein V, Blumenberg V, Ackermann J, et al. Single-center experience with axicabtagene-ciloleucel (Axi-cel) and tisagenlecleucel (Tisa-cel) for relapsed/refractory diffuse large B cell lymphoma: comparable response rates and manageable toxicity. Blood. 2020;136(Supplement 1):34-5.

71. Pasquini MC, Hu Z-H, Curran K, et al. Real-world evidence of tisagenlecleucel for pediatric acute lymphoblastic leukemia and non-Hodgkin lymphoma. Blood Adv. 2020;4(21):5414-24.

72. Jaglowski S, Hu Z-H, Zhang Y, et al. Tisagenlecleucel chimeric antigen receptor (CAR) T cell therapy for adults with diffuse large B cell lymphoma (DLBCL): real world experience from the Center for International Blood \& Marrow Transplant Research (CIBMTR) Cellular Therapy (CT) Registry. Blood. 2019;134(Supplement 1):766-766.

73. Nastoupil LJ, Jain MD, Feng L, et al. Standard-ofcare axicabtagene ciloleucel for relapsed or refractory large B cell lymphoma: results from the US Lymphoma CAR T Consortium. J Clin Oncol. 2020;38(27):3119-28.

74. Iacoboni G, Iraola-Truchuelo J, Martinez-Cibrian N, et al. Real-world evidence of tisagenlecleucel for the treatment of relapsed or refractory large B cell lymphoma. Blood. 2020;136(Supplement 1):19-21.

75. Riedell PA, Walling C, Nastoupil LJ, et al. A multicenter retrospective analysis of clinical outcomes, toxicities, and patterns of use in institutions utilizing commercial axicabtagene ciloleucel and tisagenlecleucel for relapsed/refractory aggressive B cell lymphomas. Blood. 2019;134(Supplement 1): 1599-1599.

76. Grant TS, Burns D, Kiff C, Lee D. A case study examining the usefulness of cure modelling for the prediction of survival based on data maturity. Pharmacoeconomics. 2020;38(4):385-95. 